\title{
WENDELSTEIN 7-X at the transition to assembly
}

\author{
L. Wegener and the W7-X Team \\ Max-Planck-Institut für Plasmaphysik, Euratom Association \\ Teilinstitut Greifswald, Wendelsteinstrasse 1, D-17491 Greifswald, Germany \\ Corresponding author: Tel.: +49-3834-882721; fax: +49-3834-882719 \\ E-mail address: lutz.wegener@ipp.mpg.de
}

The WENDELSTEIN 7-X (W7-X) represents the continuation of fusion experiments of the stellarator type at the Max-Planck Institute for Plasma Physics. The experiment W7-X is built at Greifswald in the northeast of Germany. The size of the device (725 t, height of approx. $4.5 \mathrm{~m}$, diameter approx. $16 \mathrm{~m}$ ), a superconductive magnet system (MS), extremely narrow construction conditions and complexly bent 3dimensional components distinguish the W7-X from earlier stellarators at the MaxPlanck-Institute. This paper gives a survey of the applied assembly technology and presents the status of the preparations.

The construction of W7-X had already been described in detail in former contributions (see [1], [2] and figure 1). The basic machine W7-X is constructed from 5 modules. Each module contains one fifth of the complete MS and a fifth of the cryostat with the plasma-facing components (PFC). The whole superconductive MS consists

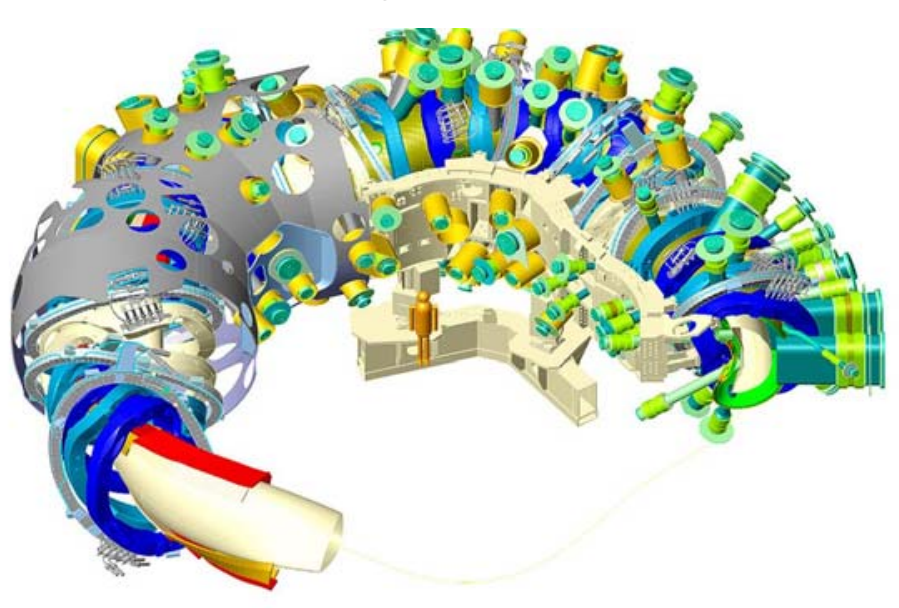

Figure 1: CAD model of W7-X of 70 coils and a complex central support structure (CSS). A coil weighs six tons and has a diameter of approx. three metres. The cryostat which envelops the MS comprises the plasma vessel (PV), the outer vessel (OV) and 299 ports as well as the thermal insulation (TI) and the cryogenic shield. The PFC contain among others the actively cooled wall protection, the divertor and 20 control coils.

A short overview of the present delivery status of the components of $\mathrm{W} 7-\mathrm{X}$ is as follows: 15 out of 70 superconducting coils have been delivered at present. The first two half-modules are being assembled. The entire plasma vessel, which consists of 20 sectors, has already been supplied. The first module of the central support structure was supplied in spring 2007 whereas the delivery of the first module of the outer vessel is planned for end of 2007 . The first sections of the thermal insulation have been mounted onto the first plasma vessel sectors. The thermal insulation for the outer vessels and for the ports is being designed respectively manufactured. The superconducting bus-bar system which is used to connect the 70 coils with each other is being produced by the FZ Jülich. The first 3D-bent conductors have been completed and successfully tested. Low resistive joints have been developed and successfully qualified and tested in SULTAN in Switzerland and in the Efremov Institute in Russia. 
The delivery of 299 ports was completed. Both the central machine base which carries the whole machine and the equipment for the final assembly stage were recently installed. The construction of the plasma facing components is in full swing, first parts have already been fabricated (wall panels, control coils). The first works on the peripheral equipment like water cooling system, low voltage power supply and helium supply system have started. Further details are represented in [3], [4]. Notwithstanding this progress there are still some assemblies (e.g. mechanical support elements) whose construction and production must be accelerated.

In the further course the contribution presents the special challenges of the assembly of the W7 X. The W7-X assembly process is subdivided into three main phases: the component preparation; the pre-assembly and the final assembly.

The preparation work belongs to the component procurement and will not be further dealt with in detail in this paper (figure 2). Main activities are the installation of sensors and instrumentation, welding work on superconductor ends, installation of the TI panels onto plasma vessel sectors mechanical adaptations, metrology and incoming inspection. Eight to ten weeks are required at present to prepare components like coils or vessel sectors completely.

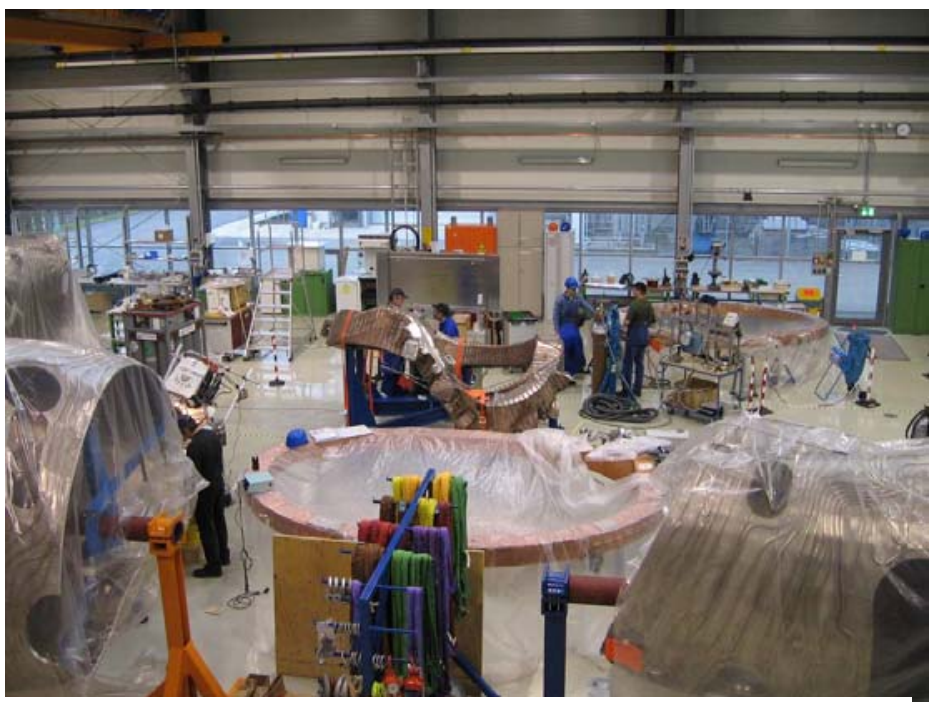

In the pre-assembly (figure 3 ) single components are put as-

Figure 2: Coils and plasma vessel sectors in the component preparation hall sembled to form half-modules (one tenth of the whole magnet system, about 50 tons) respectively modules (two connected half-modules). At first coils are being strung

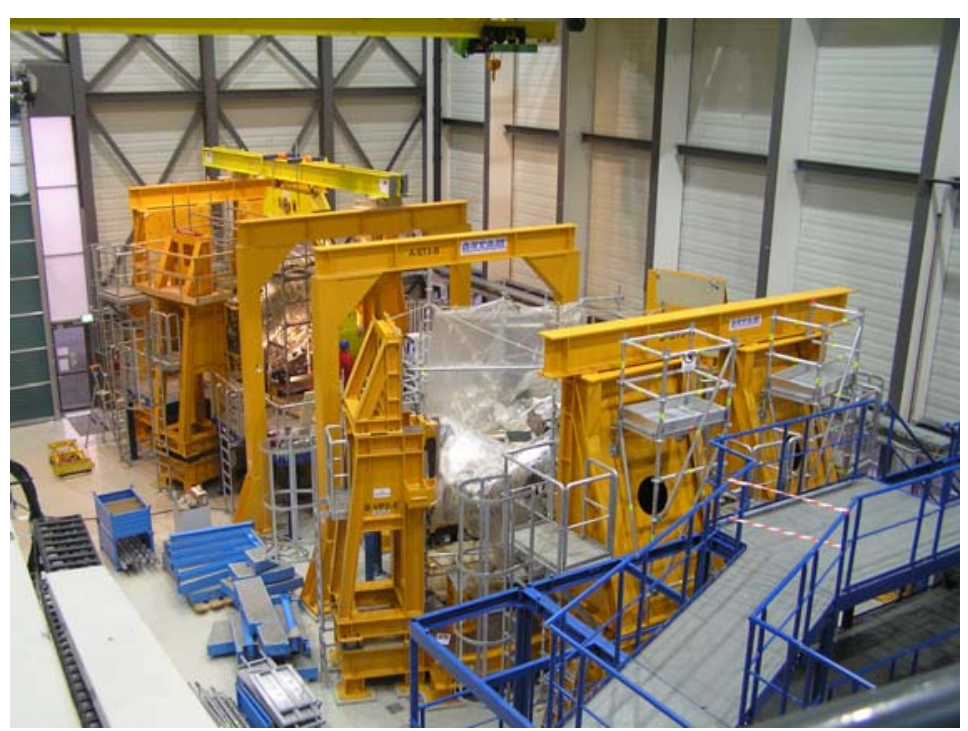

Figure 3: The pre-assembly hall where two flip symmetric half-modules are presently built up in the mounting stands la and Ib (MST la and lb). across the pre-aligned plasma vessel sectors. The clearance between coil and plasma vessel spans only few millimetres. Due to the 3Dbent geometry of coils and plasma vessel the coils needs to be shifted, tilted and rotated simultaneously in all six degrees of freedom. Heavy computer assisted tools were developed which enable that coil movement along the mathematically optimised threading path. Coil and tool are hanging in the crane and weigh about 12 tons.

The alignment of the 3Dshaped components requires the intensive use of laser tracker and photogrammetry systems. An alignment accuracy of about $1 \mathrm{~mm}$ was achieved. After seven coils have been threaded across the plasma vessel a sector of the central support ring (CSR) is bolted with the coils. 


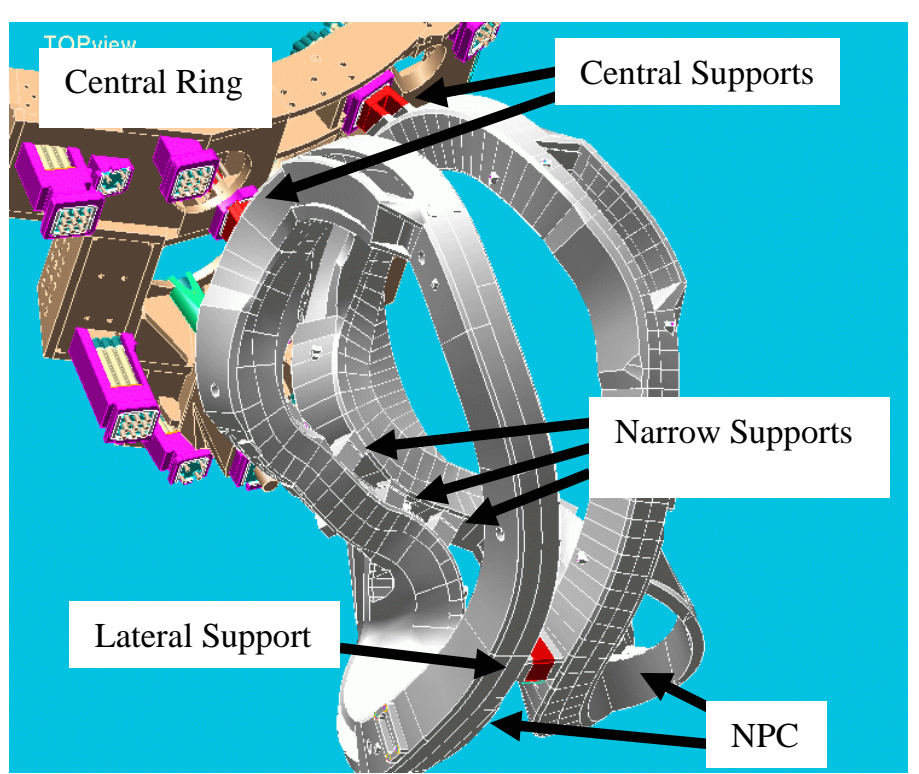

Figure 4: The position of central, lateral and narrow supports between two neighbouring coils
Every coil has got two central supports (CSE) which connect it with the CSR (figure 4). Additional support elements connect every coil with its right respectively left coil neighbour. These support elements form two cascaded circumferential support-belts. One consists of lateral support elements (LSE) which are welded together with the coils. The other one consists of narrow support elements (NSE) which make a gliding and rolling movement possible between the coils (figure 5). During the operation the magnetic forces are being distributed between CSR, CSE, LSE and NSE. High assembly accuracy is needed to ensure the calculated load distribution and to avoid an overloading of single support elements. The joint between coil and ring is complemented with several fitting elements and shims. Complex cast procedures and manufacturing procedures have been developed to enable the installation of these elements under a very restricted accessibility. Latest installation trials using an aligned coil unit together with a central support ring have proven a reliable and clashes-free mount ability of the structural parts. Lateral supports elements have to be welded between neighbouring coils to increase the mechanical stiffness of the magnet system. Welding procedures were developed that ensure a welding shrinkage inaccuracy of approximately $+/-0.5 \mathrm{~mm}$ at a weld seam thickness of more than $20 \mathrm{~mm}$. All steps of the pre-assembly of half modules have been successfully qualified. Comprehensive assembly documentation was worked out (working instructions and inspection instructions). These works already run routinely.

Two half modules are bolted to each other after both have been preassembled (flip symmetric construction). A separate mounting stand is used to align both 50 tons half-modules within a global accuracy of about $1 \mathrm{~mm}$. During the further assembly the plasma vessel sections hang in the coils. They are aligned to each other and welded together. A full penetration weld of $8 \mathrm{~m}$ circumferential length and $17 \mathrm{~mm}$ thickness is made. The contour deviation due to the welding of the vessel stays within

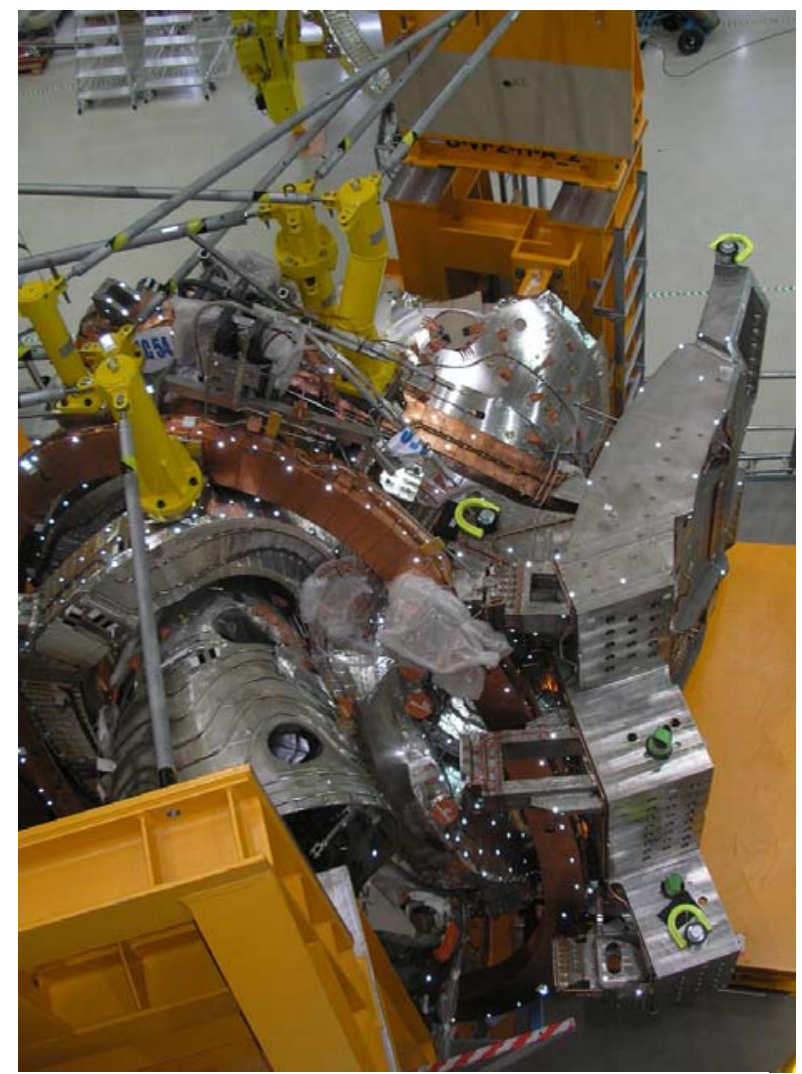

Figure 5: A half module during the assembly: the plasma vessel (partially insulated) positioned and clamped between assembly structures; three non-planar coils and two planar coils are positioned and aligned; the central support ring (CSR) has been moved and aligned in front of the coils. 
about $3 \mathrm{~mm}$. After the mechanical joining of the magnets and the plasma vessel (figure 6 ) the 14 coils are electrically and hydraulically connected with 24 singly prefabricated bus-bars (3D bent, helium tight and Paschen proved).

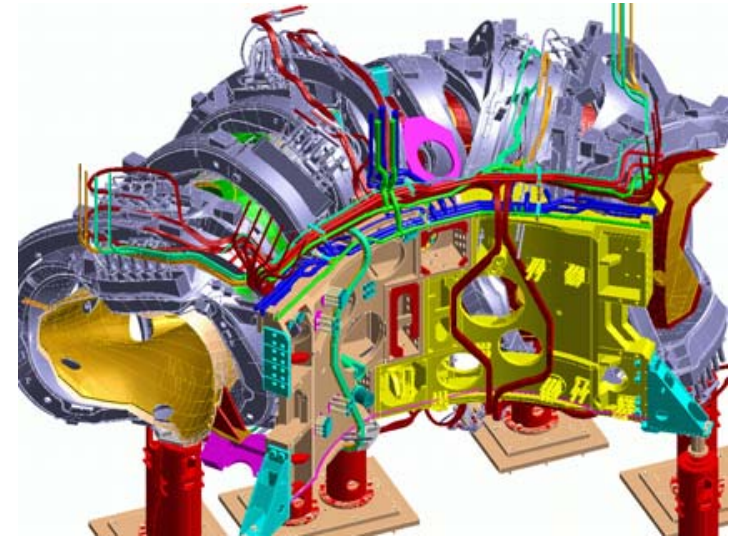

Figure 6: Two flip symmetric half-modules on mounting stand II (MST II), positioned together at the stairs-shaped flange in the middle of the CSS (one fifth of the central support ring); busbars and helium manifold are mounted
The mechanical fixation of the bus-bars is a demanding task. On the one hand the bus-bars have to follow the flexible displacements of the magnet system during the operation. On the other hand they have to be sufficiently supported to withstand the electromagnetic forces. As a consequence complex support bearings have been developed and successfully tested (figure 7). The bus-bar ends and the coil terminals are connected with dismountable electrical joints. A joint achieves a resistance of less than $1 \mathrm{n} \Omega$. It withstands pressure strokes up to 170 bar. The joints are removable to enable the repair of single coils.

The joint-housings are made of steel whereas the superconductor jacket is made of aluminium. Aluminium-steel transition pieces enable the welded connection between both components. The welding of both, the transition pieces as well as the jointhousings is a very sensitive operation since the superconductor must not be overheated. Special lip-welding procedures have been successfully developed. The bus-bar system is wrapped with a $4 \mathrm{~K}$ compatible insulation system. A test voltage of up to $13 \mathrm{kV}$ is applicable to the bus-bar system. The completely preassembled magnet-module is being transported into the experiment hall for the final assembly. A special jig (MST III) enables the movement of the magnet module through the gate of the experimental hall. The jig serves simultaneously the completion of the bus-bar as-

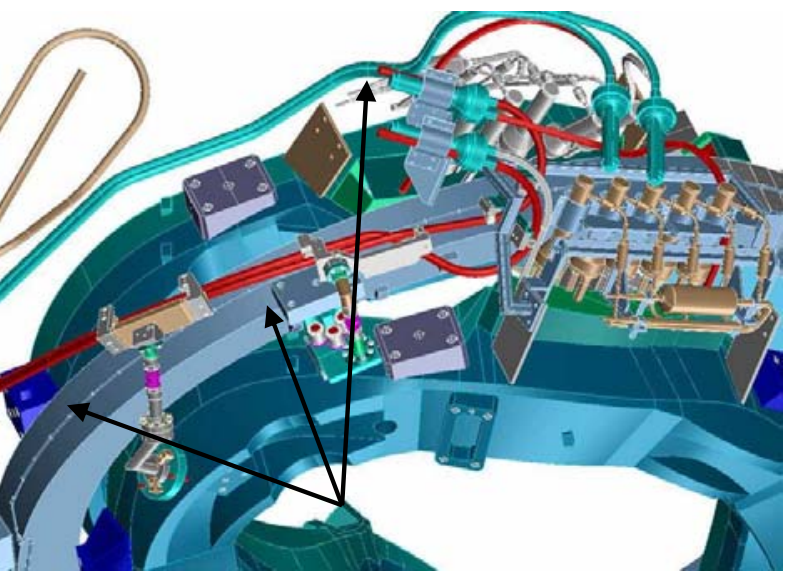

Figure 7: Mechanical supports hold the insulated bus-bars $(13 \mathrm{kV})$ in position. The supports have to partially resist high magnetic forces and on the other hand they have to flexibly take displacements.

sembly. This avoids a bottleneck in the assembly sequence since the pre-assembly of half-modules is faster than the assembly of magnet modules. A heavy lifting beam with an adjustable gravity centre under full load was developed to enable a plane movement of half-modules and modules throughout the assembly stages.

In the final assembly each of the sequentially prefabricated five modules is completed with the outer vessel (OV) (figure 8). Due to the many openings the OV needs to be stiffened with special reinforcements. The OV is cut into two shells: the lower shell and the upper shell. In a first step the plasma vessel supports (three per module) are being aligned and welded to the lower shell of the OV (MST IVa). Then the thermal insulation is installed. Two so-called cryo-feet which later carry the dead weight of the magnet module are being positioned through openings in the lower shell. The cryo-feet form the thermal break between the $4 \mathrm{~K}$ level (magnet system) and ambient temperature. Thereafter by the use of the above lifting beam the preassembled magnet system is lowered into the lower shell. The cryo-feet are bolted to 


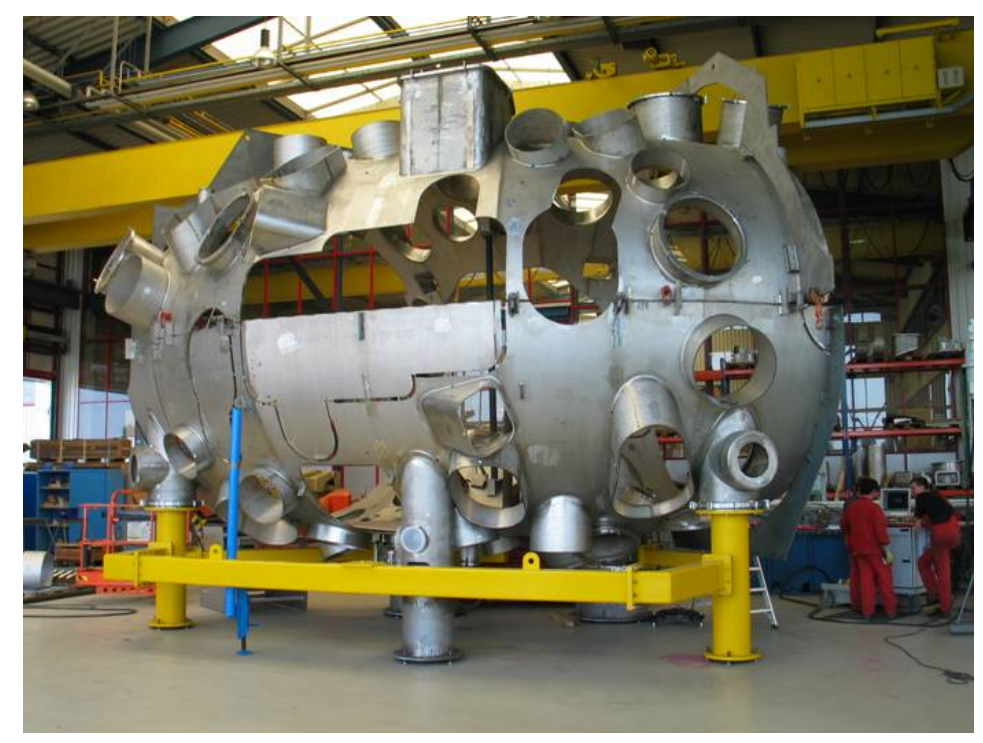

Figure 8: The outer vessel (OV) divided in the lower and the upper shell. Inner reinforcements (poles) and outer reinforcements (yellow) stabilise the vessel during the assembly.

the magnet module and bellows are welded between OV openings and cryo-feet to close the cryostat at these points.

Magnet system and OV are mechanically decoupled. Now both the magnet module (with the PV module hanging inside the coils) and the lower shell independently hanging in the lifting beam are transported onto the central machine base. All three units (MS, OV lower shell, PV) are sequentially aligned in the global coordinate system using a special alignment jig (MST IV; figure 9) and the laser tracker survey.

The PV-Module is positioned onto the plasma vessel supports which have been previously welded to the lower shell of the OV. The already prepared upper shell (with thermal insulation) is positioned above the lower shell and slowly lowered down. During this movement the thermal insulation of the lower and the upper shell are overlapped, therewith closing the thermal insulation of the OV. The above OV reinforcements are used to align the welding gap between lower shell and the upper shell. Lower shell and upper shell are welded together and inspected. With this step one module of the OV has been completed. At this stage about 50 ports are installed together with the complex thermal insulation (MST V). Ports weigh between 150 and $1500 \mathrm{~kg}$. Their alignment within a sub-millimetre range in all three axes requires also special equipment which is presently provided (figure 10).

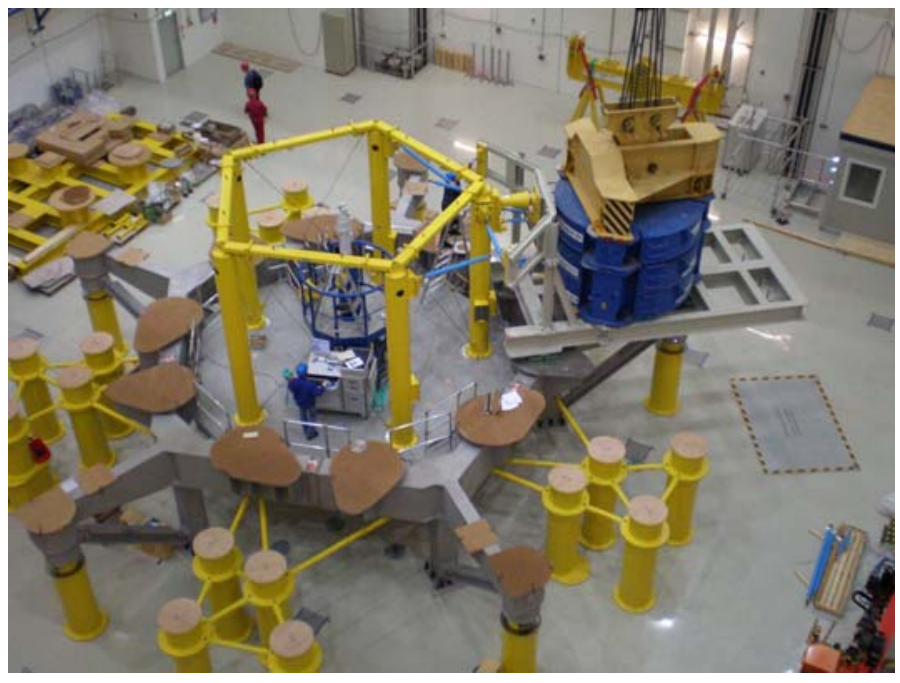

Figure 9: The mounting stand IV (yellow structure) on the machine base; alignment trial with a 100 tones dummy load. The achieved accuracy was better than $1.5 \mathrm{~mm}$.

All ports have to be cut to shape and length. Therefore a dummy is used to take up the actual 3D-contour of the opening in the PV. That contour is being transferred onto the real port which then is cut manually. A port is then precisely positioned between OV and PV and its position is surveyed. Afterwards the port is simultaneously tackwelded at both sides: from the PV inner side and from the OV outside. Due to the large number of ports and different assembly places special logistics is needed to minimise the assembly time of ports. The work of port assembly is made in parallel wherever possible. Furthermore there are several so-called special ports for which the assembly procedure must be first adapted. Due to accessibility constraints further 10 ports (of about 60 per module) can be only installed after the whole torus has been closed. After that the first section of plasma facing components is installed; 
mainly the cooling pipes, the instrumentation and mechanical brackets. The details are at present being worked out. The in-vessel installations require the comprehensive use of the metrology. A fifth of the base machine is at that point completed. Once the third module has been completed in the same way work starts to join the neighbouring modules with each other. This work includes bolting of the magnet structure, welding of the plasma vessel and the outer vessel, the connection of busbars and installation of ports and thermal insulation must be done but in a very narrow working area. After joining the fifth module, the last section of the plasma facing components is installed. This includes the very sensitive parts of the thermally highly loaded divertor. After this work the basic machine is ready for the commissioning. Parallel to the work mentioned above, the Periphery is built up. This work comprises: the cooling system, low voltage power supply, vacuum and helium system, instrumentation etc.

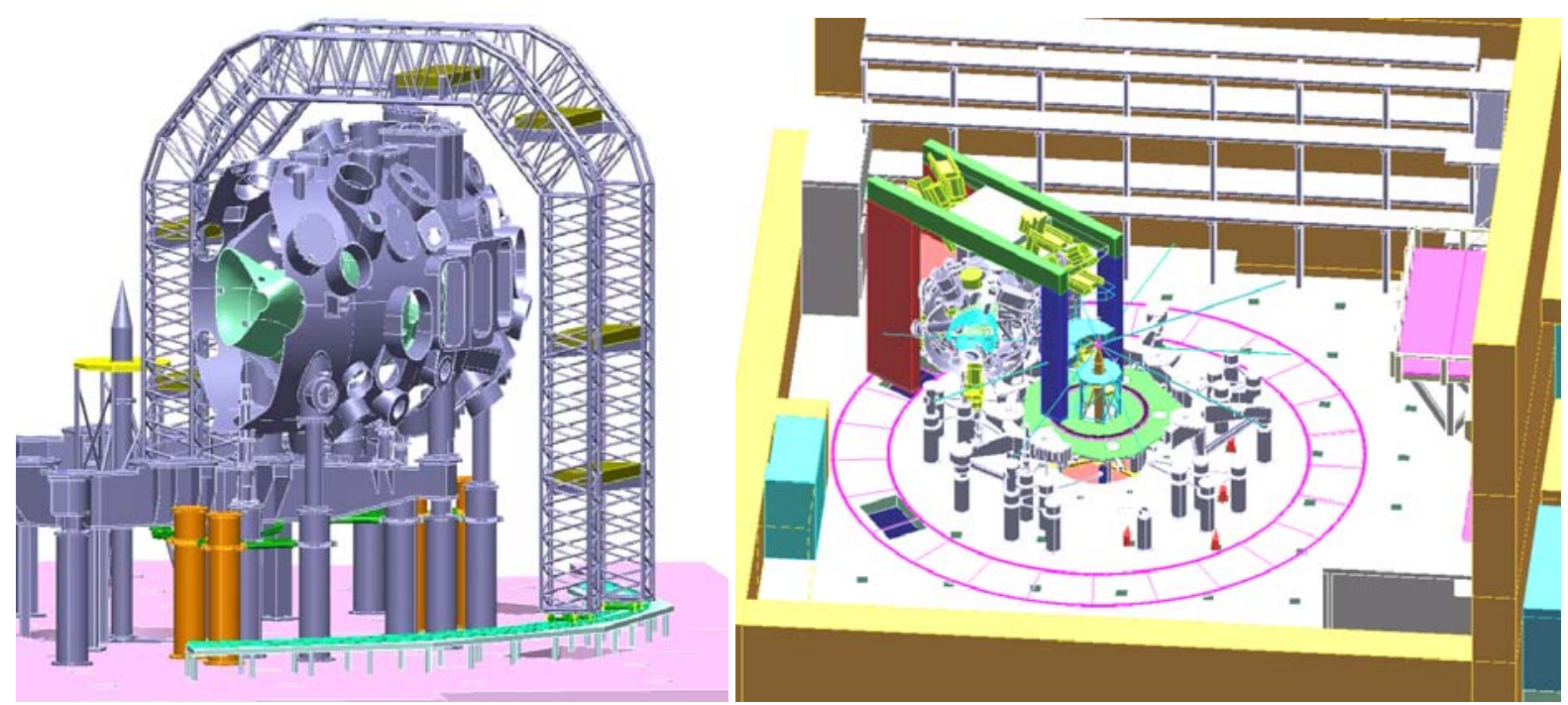

Figure 10: The planned equipment for the installation of ports. Principle:

- Align able ramps $(x, y, z$ and $\operatorname{rot}(x, y, z))$ on a movable bridge. The port is fastened on the ramp on one side via an interface flange.

- Two ramps are independently operated; a third one on the floor

- The moveable bridge runs on rails; the alignment is surveyed by an Laser Tracker positioned in the machine centre

An efficient planning system has been set-up to govern the entire assembly process. Besides the assembly technology, which describes and plans the required technical assembly steps, the actual delivery dates of the components, the very limited space in the assembly area, the tight schedule and limited resources represent the boundary conditions for the assembly planning. The schedule of W7-X assembly requires a two shift system. The majority of the needed technicians and craftsmen will be hired from industry, whereas the middle and upper management and the engineer's staff are made available by IPP staff. Quality checks will be done and documented to a large extent by the assembly staff itself. Special or expensive checks and the quality survey will be done by independent inspectors of the quality management department. The metrology team plays a essential role in the assembly of W7-X. It assists the alignment of almost all components and it checks and surveys the "As Built" geometry of W7-X.

The whole assembly process is structured by work instructions (AA) and inspection instructions (PA) that fix all details of single assembly steps. Standardised inspection sheets will be used to report inspection results. All instructions are listed in many Quality Assurance and Assembly Plans (QAAP) combining all the required working and testing steps and also listing all the hold points, signatures, and dates etc. From the present perspective about 450 single assembly sequences are needed to survey 
and to document the whole assembly until the commissioning phase. Presently it is estimated that about 700000 man hours are needed to build W7-X. Unfortunately, no special acceleration measures are available at the moment to speed up W7 $\mathrm{X}$ assembly. Therefore it is planned to thoroughly revise the existing assembly planning, based on the present knowledge. Possible measures are: a use of increased working time, the simplification of some assembly steps and the increase of some risks.

\section{References}

[1] M. Wanner, J.-H. Feist, H. Renner, J. Sapper, F. Schauer, H. Schneider, V. Erckmann and the W7-X Team, Design and construction of Wendelstein 7-X, 21st Symposium on Fusion Technology, Madrid, Spain, September 2000, paper IN-18

[2]. M. Wanner, V. Erckmann, J.-H. Feist et al., Status of WENDELSTEIN 7- Construction, Nuclear Fusion 43 (2003) 416-424

[3] F. Schauer on behalf of the W7-X team, Status of WENDELSTEIN 7-X Construction, $24^{\text {th }}$ Symposium on Fusion Technology, Warsaw, Poland, September 2006, PL2-0-561

[4] L. Wegener and the W7-X Team, WENDELSTEIN 7-X at the transition to assembly, Fusion Engineering and Design 74 (2005) 41-48 